\title{
Conservative treatment of subdural empyema: a complication of odontogenic sinusitis
}

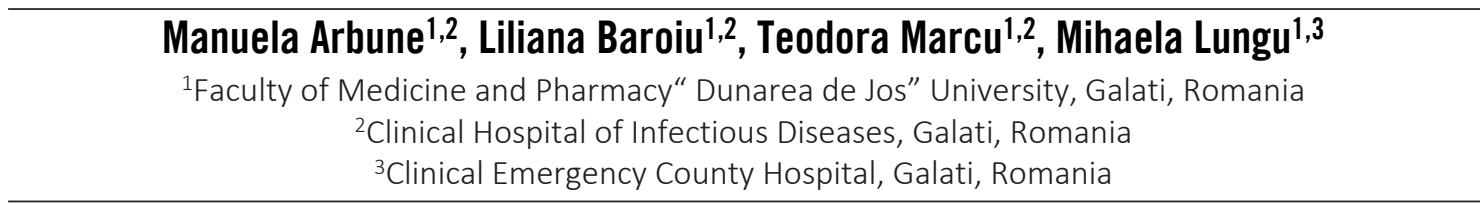

\begin{abstract}
Odontogenic infections are predisposing factors for $10 \%$ of maxillary sinusitis, rarely complicating with meningitis and subdural empyema. Most empyema associated with dental infections have polymicrobial etiology. Standard treatment for subdural empyema contains supportive care measures, antibiotic combinations and surgical drainage. We present a rare case of subdural empyema on a 20 -year-old man with neglected odontogenic infections. outcome by antibiotic conservative treatment and no neurosurgical intervention.
\end{abstract}

Keywords: subdural empyema, odontogenic infections, antibiotic treatment

\section{BACKGROUND}

Subdural empyema is a purulent collection located in the space between the dura mater and the arachnoid mater (1). Trigger factors of subdural empyema are regional infections, cranial traumas and haematogenous dissemination from an extracranial infection (2). The etiology varies depending on the geographical area and the source of infection and also the patient's characteristics such as: age, immune status and co-morbidities. The main therapeutic measures are antibiotic administration for 4-8 weeks and control of intracranial pressure. Surgical procedures such as excision and drainage are usually imperative, but those can be temporized in case of small collections, recently formed (3).

\section{CASE PRESENTATION}

A 20-year-old urban male, smoker, currently an emigrant for the purpose of the employment, pre- sents to the emergency department with frontal headache and fever, approximately 10 days before the admission. In the last 24 hours purulent rhinorrhoea and neurologic deficit of the left upper limb have been developed. Medical history points out recurrent upper respiratory infections and multiple neglected dental problems, over the past three months, for which the patient self-medicated with nonsteroidal anti-inflammatory drugs and unspecified antibiotics.

The hospital baseline clinical examination pointed out: fever $390 \mathrm{C}$, heart rate $95 / \mathrm{min}$, respiratory rate $24 / \mathrm{min}$, blood pressure $115 / 60 \mathrm{mmHg}$, left upper and lower limb paresis and meningeal syndrome, Glasgow Coma Scale 15 and qSOFA scale 0 .

Laboratory evaluation points out leukocytosis $\left(14,260 / \mathrm{mm}^{3}\right)$ and completes the four criteria of the systemic inflammatory response syndrome together with: fever, tachycardia and tachypnea. The 
common inflammatory biomarkers were positive. A sepsis complication was suggested by the increased level of procalcitonin with over $10 \mathrm{ng} / \mathrm{ml}$. Serologic tests for HIV, HBV, HCV and syphilis were negative.

The native computer tomography (CT) scan of the brain revealed right pansinusitis and left ethmoidal sinusitis, enlargement in the right parietal structure of the cortex, multiple aeric images in the left parietal and right frontal lobes and in the cerebral falx. The ventricular system was symmetric, with normal dimensions.

The lumbar puncture was achieved. The cerebrospinal fluid (CSF) was characterized by: pleocytosis $\left(450 / \mathrm{mm}^{3}\right)$ with predominance of polymorphonuclear leucocytes $(80 \%)$, increase of albumin $(66 \mathrm{mg} / \mathrm{dL})$ and low glucose $(6,52 \mathrm{mg} / \mathrm{dL})$. Bacterial menigoencephalitis was the presumptive diagnosis, although the etiology was unspecified. The microscopic exam and the aerobic bacterial cultures were negative. Culture media for anaerobic bacteria were not available.

The first line antibiotic treatment was Meropenem, but the clinical evaluation after 72 hours pointed out persistent fever, aggravation of the neurological status with 8 points on Glasgow Coma Scale and left hemibody seizures. Reevaluations by the magnetic resonance imaging (MRI) revealed right sphenoidal, frontal and maxillary sinusitis and also an image with 2,5 $\mathrm{mm}$ - thickness, indicative of a right anterior parietal empyema (Fig. 1).

The neurosurgical procedure of empyema drainage was analyzed by the medical team and was decided to be temporized. The antibiotic therapy was reconsidered replacing Meropenem in combination with Ceftriaxone, Vancomicine and Metronidazole and the evolution turned out positive after a 21-day. The patient was discharged on request but he came back for a scheduled checkup after one month, when the clinical examination revealed normal findings, except the unsolved dental problems (Fig. 2).

\section{DISCUSSIONS}

The subdural empyema can develop complications in $2 \%$ of adults with bacterial meningitis (4) and represents $20 \%$ of the focal intracranial suppurations in neurosurgical praxis. The prevalence of subdural empyema is higher in males and during the second and third decades of life, concordant with the higher frequency of otitis and sinusitis occurring at these age (5).

A meta-analysis on 51 studies which evaluated the etiology of the localized cerebral suppurations
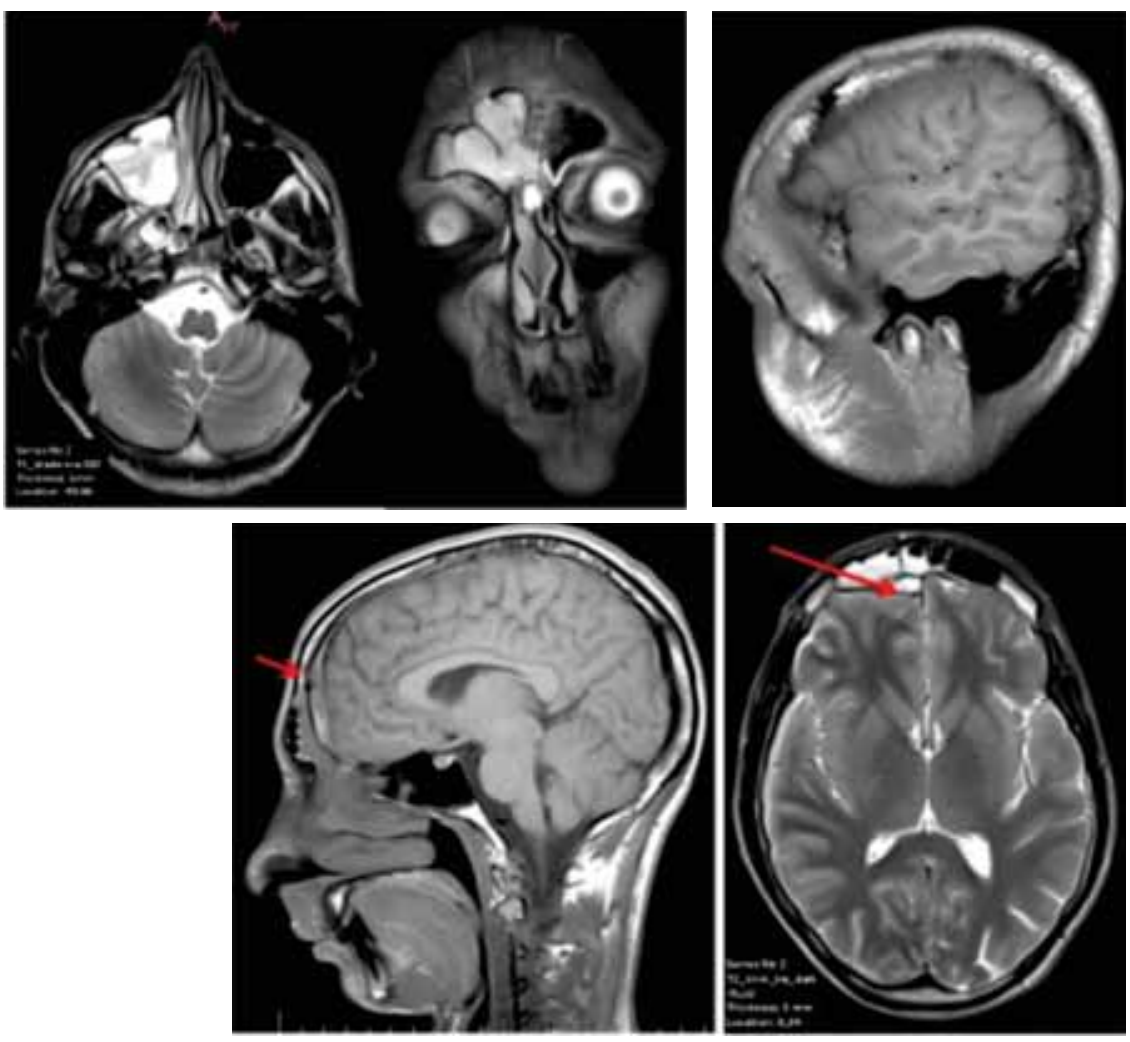

FIGURE 1. IMR of thebrain ( $a, b, c, d, e)$ Accumulation in the hypersignal area on T2-weighted images and the hyposignal area on T1-weighted image, with restriction on the diffusion sequence, located in the subdural layer, on the right fronto-parietal area, with a maximum thickness of $2.5 \mathrm{~mm}$; sereveals strong enhancement and a thickened aspect of the convexitary meningeal structures on the right side after the administration of contrast medium; no signal-intensity changes in the supra and infratentorial cerebral parenchyma on native and post-contrast administration; normal size of the ventricular system; the thickened mucosa with hypersignal on T2-weighted images on the right, sphenoidal and frontal maxillary sinus, which associates accumulations in the hypersignal on T2-weighted images on the right and frontal maxillary sinuses. 


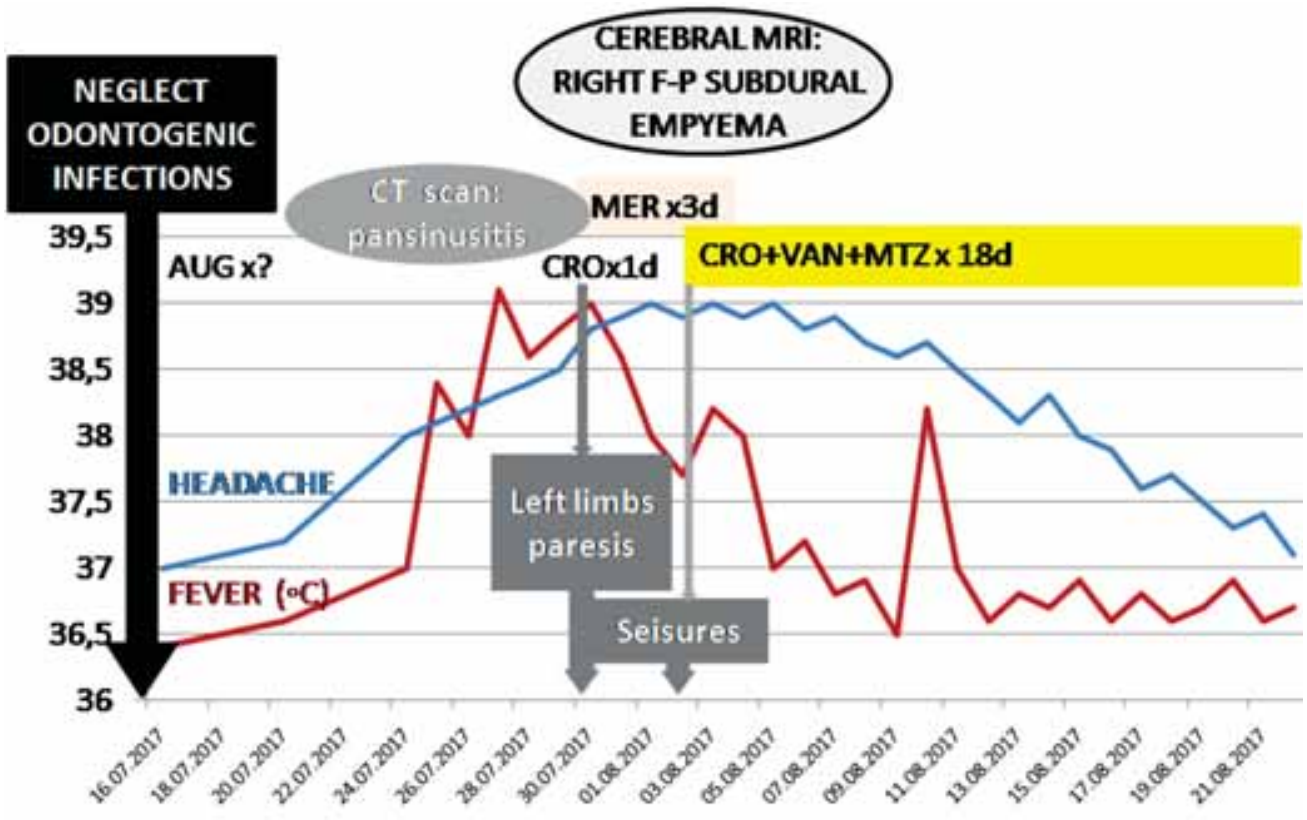

FIGURE 2. The diagram of subdural empyema evolution and treatment in a 20-year-old male patient

Legend: MER: Meropenem; CRO: Ceftriaxone; VAN: Vancomicine; MTZ: Metronidazole;

F-P: fronto-parietal; CT: computer tomography; MRI: magnetic resonance imaging; d: days.

has analyzed 2,653 bacterial cultures of the cerebrospinal fluids, from which $30 \%$ were found negative and $26 \%$ were polymicrobial (6). However, the frequency of polymicrobial etiology seems to be higher, with an estimated rate over $50 \%$ from the brain suppurations. The main isolated bacterial species were: Streptococcus spp (36\%), Staphylococcus spp (17\%), Enterobacter spp (12\%), Bacteroides spp (7\%), Haemophilusspp (3\%), Peptostreptococcusspp (3\%), others (17\%) (4). Anaerobic bacteria are involved in the majority of brain suppurations, mainly related to those with odontogenic origin, their isolation rate is low, due to the difficulties to ensure adequate transportation conditions of anaerobic cultures (7). The etiologic identification rate increases over three times in the case of the analysis of the aspirate in empyema using the molecular technique of 16S rRNA gene for bacteria, but this procedure is not routinely available yet (8).

The etiology of rhinosinusitisis different from the one of the odontogenic sinusitis. At the beginning of the case history, the native CCT exam revealed maxillary sinusitis, that was associated with purulent meningitis, explained by the common etiology with Streptococcus pneumoniae, Haemophilus influenzae and Moraxella catarrhalis. The first line antibiotic therapy with Meropenem was decid- ed considering the antibacterial broad spectrum, the ability to penetrate the blood-brain barrier, as well as the favorable safety profile. However, the treatment proved to be ineffective due to the subdural empyema, that was identified in the imaging examination after a 3-day evolution. This situation is explained together with the highlighting of the dental problems and the dental history which lead to the odontogenic origin of sinusitis. The characteristics of odontogenic sinusitis is mixed etiology (aerobic and anaerobic germs) and the frequent association of Bacteroides spp, with common Carbapeneme resistance(9). Seizures are part of the common clinical picture of meningoencephalitis, although it might be also a side effect of Meropenem, but this was only described in $0.08 \%$ of the patients (10). The evolution of seizures was favorable with anticonvulsant treatment (Carbamazepine). However, continuous monitoring and longterm neurological treatment are recommended.

Anatomically, the upper molar roots are situated on the floor of the upper jaw maxilar sinus, allowing fistulisations and the extension of the infection from the upper dental roots infections, especially from the second molar to the maxillary sinuses and then to the cerebral tissue.

The guidelines for the treatment of CNS suppurative infections are recommending antibiotic as- 
sociation of Ceftriaxone, Vancomicine, and Metronidazole, considering the efficient blood-brain barrier penetration and the antibiotic susceptibility of germs from the dental infections, as anaerobic bacilli, enterobacteriaceae, Gram positive cocci, as well as drugs Streptococcus pneumoniae or Staphylococcus spp with problems of resistance.

The native CT exam performed in emergency might be false negative for the diagnosis of the new brain collections, in the first few days (11) so, it is necessary to repeat the imaging investigations, as it can be noticed in our case. The mass effect is especially determined by edema and ischemia, less by abscess. Although the native CCT did not reveal localized cerebral injury, the presence of aeric images in the frontal and parietal right areas and the left limb paresis might have been significant for sinusitis with anaerobic germs and bone erosions, predicting the risk of empyema.The thickness of the empyema in our case had $2.5 \mathrm{~mm}$ and was treated with antibiotics, without any surgical procedure. Conservative treatment can only be performed in empyaema which are smaller than $1.5 \mathrm{~cm}$ (12), but this option is rarely preferred (13). The aspiration procedure with microbiological identification from the aspirate was not available in our center, first choice of antibiotic therapy being based on clinical judgment and therapeutic guidelines. The optimal duration of the antibiotic treatment for subdural empyema is not specified, but most recommendations indicate at least a 4-8 weeks therapy, depending on its evolution $(1,4)$. Global mortality in cases of subdural empyema vary between 15 and $25 \%$. The evolution of empyema with odontogenic source proved to be favorable in our case, but late complications after intracerebral infection may occur, for which the patient needs to be monitored. Complex dental treatment is required for the drainage of complicated odontogenic infections in order to prevent further relapses.

\section{CONCLUSIONS}

The empirical treatment of brain suppurations developed due to odontogenic infections should include active antibiotics for anaerobic germs. The indication for conservative antibiotic treatment depends on the dimensions, location of the lesions and the biological status of the patient. Early diagnosis of subdural empyema is crucial for favorable prognosis.

\section{REFERENCES}

1. Harrison CJ. Focal Suppurative Infections of the Nervous System. In: Long SS, Pickering LP, Prober CG editors. Principles and Practice of Pediatric Infectious Diseas. 4th ed. Pennsylvania: Churchill Livingstone; 2012: 319-330.

2. Honda $\mathrm{H}$, Warren DK. Central nervous system infections: meningitis and brain abscess. Infect Dis Clin North Am, 2009; 23(3):609-23.

3. Brook I. J Microbiologyandtreatment of brainabscess. Clin Neurosci, 2017; 38:8-12.

4. Brouwer MC, Heckenberg SGB, van Well GTJ et al. from Dutch Working Party on Antibiotic Policy (SWAB), 2012. SWAB Guidelines on Antibacterial Therapy of Patients with Bacterial Central Nervous System Infections, UniversitairMedisch Centrum St Radboud, Nijmegen, 2012

5. Mandell GL, Bennett JE, Dolin R, eds. Mandell, Douglas, and Bennett's Principles and Practice of Infectious Diseases. 8th ed. Philadelphia, PA: Churchill Livingstone, 2014.

6. Brouwer MC, Tunkel AR, van de Beek D. Epidemiology, diagnosis, and antimicrobial treatment of acute bacterial meningitis. ClinMicrobiol Rev 2010; 23: 467-92.

7. KaraD, Arslan Z, Özmen O, Kara SS. Complicated Central Nervous System Infection Developing In Association with Dental Abscess in a Child. Sch J Med Case Rep 2015; 3(11):1092-1094.

8. Al MM, Armougom F, Scheld WM et al. The expansion of the microbiological spectrum of brain abscesses with use of multiple $16 \mathrm{~S}$ ribosomal DNA sequencing. Clin Infect Dis 2009; 48: 1169-78.

9. Brook I. Sinusitis of odontogenic origin. Otolaryngol Head Neck Surg 2006, 135:349-355.

10. Norrby SR, Gildon KM. Safety profile of meropenem: a review of nearly 5,000 patients treated with meropenem. Scand J Infect Dis 1999; 31:3-10.

11. Weisberg L. Subdural empyema. Clinical and computed tomographic correlations. Arch Neurol 1986, 43:497-500.

12. Greenlee JE. Subdural empyema. In: Mandell GL, ed. Principles and Practice of Infectious Diseases. Vol 1. 4th ed. New York: Churchill. 1994:900-903.

13. Brouwer MC, van de Beek D. Management of bacterial central nervous system infections. HandbClin Neurol. 2017. 140:349-364. 